\title{
Epistatic interactions of AKT1 on human medial temporal lobe biology and pharmacogenetic implications
}

\author{
HY Tan, AG Chen, Q Chen, LB Browne, B Verchinski, B Kolachana, F Zhang, J Apud, \\ JH Callicott, VS Mattay and DR Weinberger \\ Clinical Brain Disorders Branch, Genes, Cognition and Psychosis Program, Division of Intramural Research Programs, \\ National Institute of Mental Health, Bethesda, MD, USA
}

\begin{abstract}
AKT1 controls important processes in medial temporal lobe (MTL) development and plasticity, but the impact of human genetic variation in $A K T 1$ on these processes is not known in healthy or disease states. Here, we report that an AKT1 variant (rs1130233) previously associated with AKT1 protein expression, prefrontal function and schizophrenia, affects human MTL structure and memory function. Further, supporting AKT1's role in transducing hippocampal neuroplasticity and dopaminergic processes, we found epistasis with functional polymorphisms in BDNF and COMT-genes also implicated in MTL biology related to AKT1. Consistent with prior predictions that these biologic processes relate to schizophrenia, we found epistasis between the same AKT1, BDNF and COMT functional variants on schizophrenia risk, and pharmacogenetic interactions of AKT1 with the effects on cognition and brain volume measures by AKT1 activators in common clinical use-lithium and sodium valproate. Our findings suggest that AKT1 affects risk for schizophrenia and accompanying cognitive deficits, at least in part through specific genetic interactions related to brain neuroplasticity and development, and that these AKT1 effects may be pharmacologically modulated in patients.

Molecular Psychiatry (2012) 17, 1007-1016; doi:10.1038/mp.2011.91; published online 26 July 2011
\end{abstract}

Keywords: BDNF; COMT; hippocampus; lithium; schizophrenia; valproate

\section{Introduction}

The protein kinase, AKT1, is an integral node in multiple signaling cascades related to cell growth, differentiation and plasticity. AKT1 has been implicated in several key neurodevelopmental pathways affecting neuronal morphology and development, ${ }^{1}$ and may interface with the effects of dopamine (DA) and neurotrophic factors in its influence on hippocampal structure and function. ${ }^{2-6}$ AKT1 genetic variation has been associated with schizophrenia, ${ }^{7-11}$ and with fronto-striatal mediated cognitive task performance and associated prefrontal structure and function. ${ }^{11,12}$ Aberrant development and plasticity of the medial temporal lobe (MTL) is also critically implicated in schizophrenia and may involve genes controlling these processes, ${ }^{13,14}$ but the mechanistic role of AKT1 genetic variation on MTL biology in healthy and disease states is unknown.

Correspondence: Dr DR Weinberger, Clinical Brain Disorders Branch, Genes, Cognition and Psychosis Program, National Institute of Mental Health Intramural Research Program, Rm. 3C-103A, 10 Center Drive, Bethesda, MD 20892, USA.

E-mail: weinberd@mail.nih.gov

Received 19 April 2011; revised 24 May 2011; accepted 1 June 2011; published online 26 July 2011
Here, we engaged an hypothesis driven and deductive research strategy based on functional genetic variants as proxies for experimental perturbations in specific genes, which are then explored in human biology using structural and functional neuroimaging, ${ }^{15}$ as well as in conjunction with clinical pharmacological treatments that affect AKT1. We examined the MTL effects of the AKT1 rs1130233 synonymous coding variant, which has been previously linked to differential AKT1 protein expression levels, ${ }^{11,12,16,17}$ and also impacts frontal brain structure and function. ${ }^{11,12,18}$ We further examined how AKT1 variation may transduce overlapping pathways of DA signaling ${ }^{19}$ and neurotrophinmediated developmental plasticity ${ }^{1,20}$ through interactions with candidate genes implicated in these processes. Specifically, AKT1 signaling is important in the actions of BDNF in neuroplasticity and longterm potentiation in hippocampal neurons. ${ }^{21,22} \mathrm{BDNF}$ also has been thought to have a key role in the brain developmental problems associated with schizophrenia. ${ }^{2324}$ AKT1 has been shown to mediate DA signaling via a non-cyclic AMP linked pathway, ${ }^{19}$ and DA signaling is important for hippocampal learning and memory ${ }^{25}$ and prominently implicated in the pathogenesis of schizophrenia. ${ }^{26}$ Thus, we examined whether AKT1 effects were further 
dependent on genetic variation in BDNF (that is, Val66Met) and in COMT (that is, Val158Met)functional variants implicated, respectively, in dendritic BDNF trafficking ${ }^{27,28}$ and cortical DA bioavailability. ${ }^{29-31}$

Finally, in an effort to further translate these putative AKT1 brain mechanisms in humans into clinically relevant phenomena in patients with schizophrenia, we examined if higher order AKT1, BDNF and COMT genetic interactions contributed to overall genetic risk for schizophrenia, and if clinically relevant activators of the AKT1 pathway-that is, lithium or valproate ${ }^{32,33}$-modulated illness-related cognitive function and associated brain structure in pharmacogenetic interactions. Our data support the importance of these genetic interactions at multiple levels of clinical neurobiology related to human MTL biology, psychosis and its treatment.

\section{Subjects and Methods}

Subjects, cognitive testing and genotyping

Research subjects were healthy controls and patients with schizophrenia ascertained as part of the Clinical Brain Disorders Branch Sibling Study. ${ }^{30}$ Distinct subsets of these individuals were included in the functional imaging, structural imaging and pharmacogenetics sections of this study. None of these subjects have previously been studied with respect to the genetics of MTL biology reported here. In the genetics analyses, we included only subjects of European ancestry. DNA was extracted from whole blood using standard procedures. All genotypes were determined using the $5^{\prime}$-exonuclease Taqman assay. Further details of clinical ascertainment, exclusion criteria, genotyping and cognitive testing are in the Supplementary Methods. The study was approved by the NIMH Institutional Review Board.

\section{Functional imaging of memory function}

To examine memory-dependent MTL function, functional magnetic resonance imaging (MRI) data were acquired from a group of 96 healthy individuals as they performed a memory encoding and retrieval task that was previously shown to robustly engage MTL function. ${ }^{34}$ For each of the encoding and retrieval sessions, eight blocks of scenes selected from the International Affective Picture System were presented. Subjects were scanned in a 3-T MRI scanner. Imaging data and their genetic relationships were analyzed using SPM2 software (Wellcome Department of Cognitive Neurology, London; http://www. fil.ion.ucl.ac.uk/spm). Further details of the imaging task and analyses are in the Supplementary Methods.

\section{Structural imaging}

To examine MTL brain structure, MRI images from 171 healthy subjects, distinct from those in the functional imaging study, were acquired in a 1.5-T scanner. These images were processed and analyzed using optimized voxel-based morphometry with customized templates as previously described ${ }^{35}$ and detailed in the Supplementary Methods.

\section{Genetic association with schizophrenia}

We examined AKT1 rs1130233, BDNF Val66Met and COMT Val158Met genetic association for schizophrenia using a case-control data set from the Clinical Brain Disorders Branch comprising 282 unrelated patients with schizophrenia and 329 healthy controls who were successfully genotyped. A previous analysis of this data showed nominal AKT1 association with schizophrenia. ${ }^{11}$ In the present re-analysis in which we studied epistasis of these three variants, we assumed a dominance model, as in the imaging data, to avoid sparse cell sizes. The main and interaction effects of the genotypes were analyzed using a full logistic regression model, controlling for gender. Nominal significance was set at $P<0.05$ based on hypotheses about these functional genetic variants, and evidence from the imaging data. A similar analysis was then carried out for an independent replication in the GAIN (Genetic association Information network) cohort from the US (936 cases and 1190 controls; public release http://dbgap.ncbi.nlm.nih. gov/). Note though that the AKT1 single nucleotide polymorphism (SNP) typed here was rs2494731, which is in the same haplotype block as rs1130233 $\left(r^{2}>0.8\right)$ in our samples according to the Centre d'Etude du Polymorphisme Humain (CEPH) data in Hapmap (http://hapmap.ncbi.nlm.nih.gov/). Permutation tests were conducted over 1000000 random label changes within these two data sets to identify the combined empirical rate at which a set of 3-way interaction effects with the same relative risk relationships occurred at these statistical thresholds.

\section{Pharmacogenetic effects on cognition and brain} structure

In examining AKT1 effects on cognition and brain structure, we studied 186 patients who met a DSM-IV diagnosis of schizophrenia. These patients had complete pharmacological data available, were clinically stable for at least 3 months, and had cognitive data from the CBDB/NIMH patient sample above. A subset of 138 patients with structural MRI data was studied in the imaging component. These cognitive and imaging pharmacogenetic analyses have not previously been performed for these data. Illnessassociated cognitive change was estimated from the difference between premorbid IQ (from the WideRange Achievement Test reading score) and present IQ (from the Wechsler Adult Intelligence Scale). ${ }^{36}$ Decreases in various aspects of cognition associated with illness are a well-documented characteristic of patients with schizophrenia, ${ }^{36}$ are a reasonable proxy of general cognitive concomitants of illness and predict treatment outcome and disability. ${ }^{37}$ Further, we studied the pharmacogenetic effects of AKT1 on a general cognitive measure given predictions from this study, and previously, that AKT1 affects cognitive domains constituting general intellectual function. In 
particular, AKT1 effects occurred at episodic memory in this study, as well as in prior studies of working memory, executive function and processing speed, ${ }^{11}$ attentional control ${ }^{12}$ and verbal learning. ${ }^{18}$ Thus, to encompass these diverse cognitive associations and to minimize repeated testing, we chose the integrative cognitive measure represented by IQ as the most parsimonious proxy of these variable cognitive effects. Differences across patients with or without mood stabilizer (lithium or sodium valproate) were explored using $t$-tests. Relationships between cognitive change and potential confounders of gender, antipsychotic dose, duration of illness, and positive and negative symptom score (PANSS) symptom severity were explored with $\chi^{2}$ tests or Pearson's correlations. A full two-way analysis of variance was then utilized to examine the hypothesized AKT1 pharmacogenetic interaction with mood stabilizer use on cognitive change. Nominal significance was set at $P<0.05$. The hypothesized pharmacogenetic interaction was Bonferroni corrected for the 12 tests performed.

In further examining the pharmacogenetic effects on brain structure, we examined a subset of 138 schizophrenia patients with available MRI data. The MRI data were acquired, processed and analyzed similarly as described above, and in the Supplementary Methods.

\section{Results}

\section{AKT1 influences memory-dependent hippocampal} activity

Healthy human subjects $(n=96)$ engaged in our episodic memory task during functional MRI. Across genotypes of AKT1 rs1130233 (48 major allele G/G and 48 A carriers), BDNF Val66Met (61 major allele $\mathrm{Val} / \mathrm{Val}$ and 32 Met carriers) and COMT Val158Met (25 Val/Val and 68 Met carriers), there were no differences in gender, age, education, IQ, and importantly, behavioral performance indices (accuracy or reaction time) $(P>0.22$; Supplementary Tables $S 1$ and S2). Since the genotype groups did not differ in task performance, differences in the functional MRI measures of brain activity reflect changes in neural information processing, and not task performance per se.

We examined the effect of AKT1 rs1130233 during implicit encoding of complex visual scenes. We found a main effect of this variant on hippocampal activity $\left(P<0.05\right.$ corrected for false discovery rate; ${ }^{38}$ Figure 1a), where individuals carrying the A-allele associated with reduced AKT1 expression ${ }^{11,12,16,17}$ had relatively increased activation, reflecting inefficient neural processing without performance advantage.

In further assessing the functional significance of this AKT1 effect on hippocampal physiology, we examined the hypothesized role that activityregulated BDNF secretion might play in concert with $\mathrm{AKT} 1,{ }^{21,22}$ by testing for interactions with

BDNF indexed by its Val66Met functional variant. ${ }^{27,28}$ We found that orthogonal parameter estimates extracted from the hippocampal brain region showing the peak AKT1 main task effect also evidenced a significant AKT1-by-BDNF interaction $(P<0.05$; Figure 1b). In the context of the BDNF-Met allele carriers associated with relatively reduced BDNF trafficking to dendrites and reduced regulated secretion, ${ }^{27,28}$ there was an accentuated effect of the deleterious AKT1 A-allele. Next, given the important modulatory role DA plays in hippocampal memory function and synaptic plasticity in basic ${ }^{25,26,39,40}$ and human models, ${ }^{41-44}$ we further conditioned the AKT1-BDNF interaction on COMT Val/Met at this same hippocampal location to examine the modifying effect of differential DA availability predicted by this functional variant in COMT. ${ }^{29-31}$ This resulted in an AKT1-by-BDNF-by-COMT interaction $(P<0.05$; Figure 1c). Here, the AKT1 rs1130233 A-allele carriers again had relatively increased hippocampal activity; this activity was again accentuated by the BDNF-Met allele, but also by the COMT-Val homozygotes indexing increased COMT enzyme activity and relatively reduced synaptic DA. Permutation tests within this sample to identify the occurrences of sets of similar main, 2-way and 3-way interaction effects at these thresholds anywhere within bilateral MTL regions-ofinterest confirmed that the conservative rate of false positivity was $P<0.001$. These results are consistent with and in the specific direction predicted by the biological effects of these genes on hippocampalmediated plasticity related to memory encoding.

\section{AKT1 influences MTL gray-matter volume}

The hypothesis that AKT1 rs1130233 genotype would also impact on measures of MTL gray-matter volume was examined using optimized voxel-based morphometry in an independent sample of 171 healthy subjects (103 AKT1 rs1130233 G/G individuals and 68 A carriers; 111 BDNF Val/Val and 54 Met carriers; 126 COMT-Met carriers and $45 \mathrm{Val} / \mathrm{Val}$; Supplementary Table S1). Here, as predicted, the AKT1 rs1130233 A-allele was associated with loci of relatively reduced gray-matter volumes in bilateral MTL regions (Figure 2a; $P<0.001$ uncorrected).

Then, we studied if BDNF Val66Met genotype modulated the AKT1 effect on brain structure. Extracted parameter estimates from the MTL brain region with the peak main AKT1 effect did not show a significant interaction with BDNF Val66Met $(P>0.7)$, suggesting that this functional coupling mechanism might be less salient in determining MTL structure than in specific memory encoding function earlier. Next, we examined the hypothesis that AKT1 variation might affect MTL volume in epistasis with DA bioavailability indexed by COMT, given the dependencies linking AKT1 and DA levels in neurodevelopment ${ }^{4}$ and previous findings of such an interaction at prefrontal cortex. ${ }^{11}$ Similarly, we extracted graymatter volume estimates from the loci with the peak main AKT1 effect on each side, and conditioned the 
a
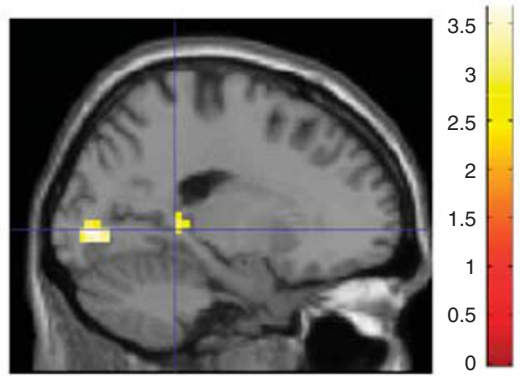

b

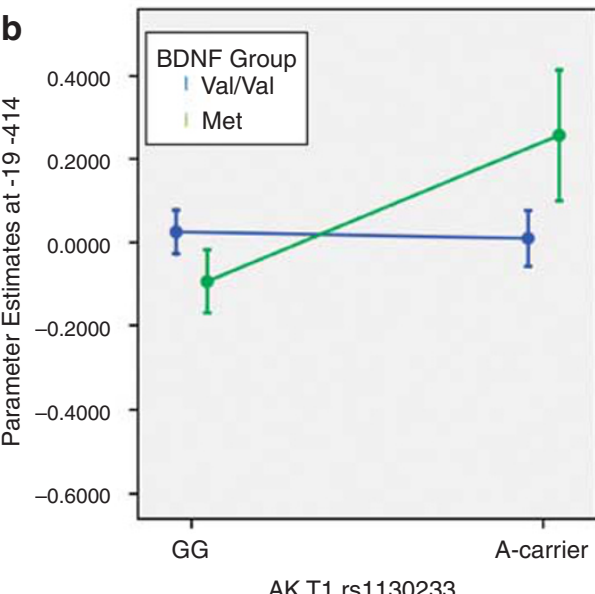

AKT1 rs1130233

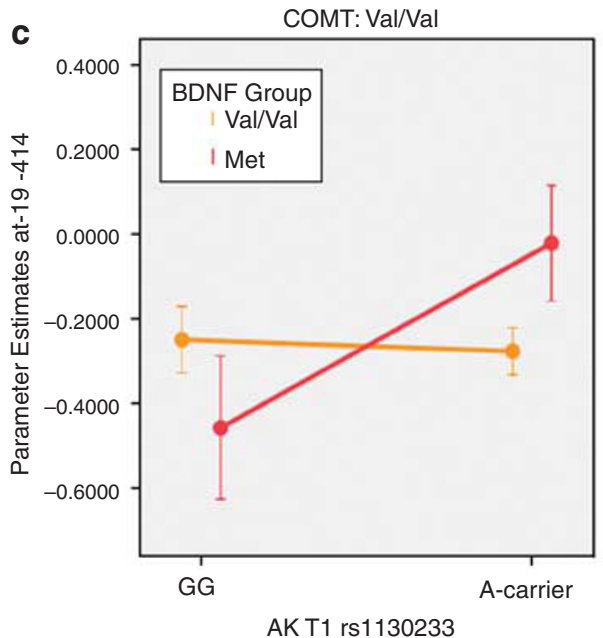

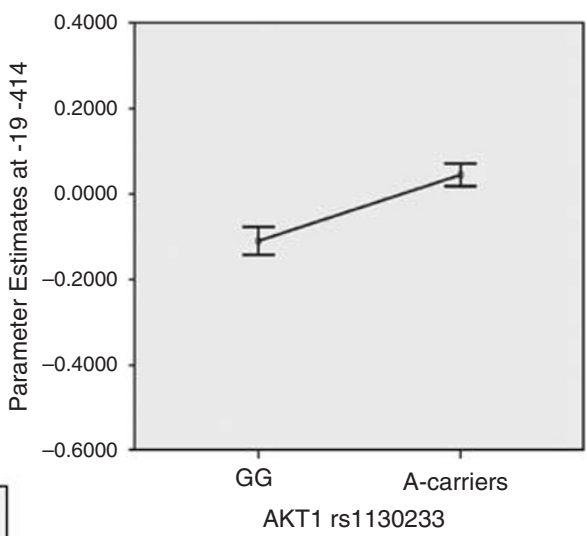

COMT: Met-carrier

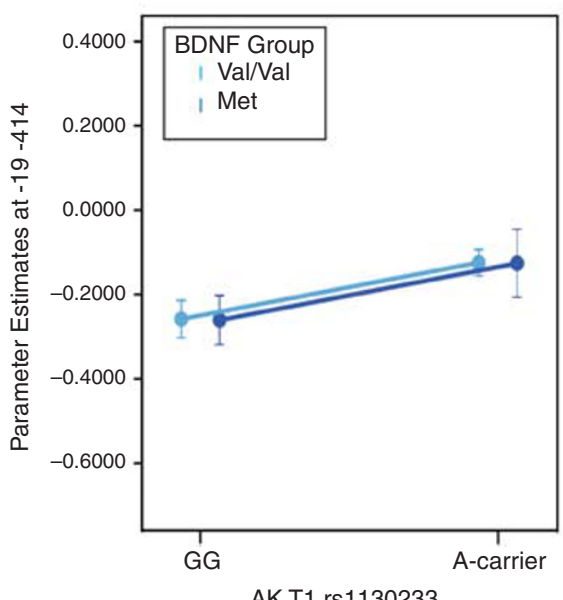

Figure 1 AKT1 and memory-dependent hippocampal activity. (a) Main effect of the AKT1 rs1130233 variant on hippocampal activity during implicit encoding of complex visual scenes (coordinates $-19-414 ; n=96, t=3.66, P<0.05$ corrected within the medial temporal lobe (MTL) region-of-interest), where individuals carrying the A-allele had relatively increased activation compared with G homozygotes. (b) Extracted parameter estimates from the hippocampal brain region showing the main task effect also evidenced AKT1-by-BDNF interaction $(F(1,92)=4.70, P=0.033)$. Individuals carrying the BDNF-Met allele $(n=32)$ had an accentuated AKT1 A-carrier effect $(n=15)$. (c) Further conditioning the AKT1-BDNF interaction on COMT Val/Met at this same hippocampal location resulted in an AKT1-by-BDNF-by-COMT interaction $(\mathrm{F}(1,90)=4.77, P=0.032)$. Here, the AKT1 A-allele effect was accentuated in individuals who were COMT-Val homozygotes and carrying the BDNF-Met allele $(n=9)$, relative to effects in BDNF-Val homozygotes and/or COMT-Met carriers. Error bars are \pm 1 s.e.

orthogonal analyses on the COMT variant. We found that $A K T 1$-by-COMT interactions occurred bilaterally $(P<0.05 ;$ Figure 2b). Individuals with combined AKT1 minor alleles and COMT-Val homozygotes associated with putatively reduced AKT1 expression and reduced brain DA, respectively, had disproportionately reduced gray-matter volumes at these loci. Permutation tests were performed to estimate the 
a

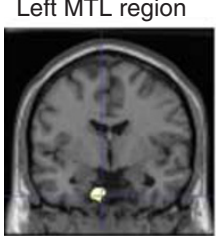

b
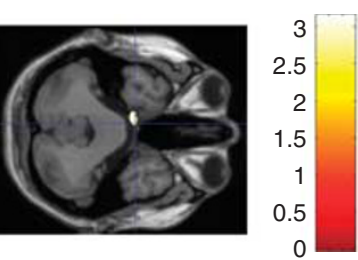

Right MTL region
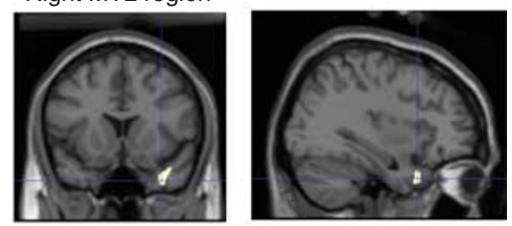

COMT genotype
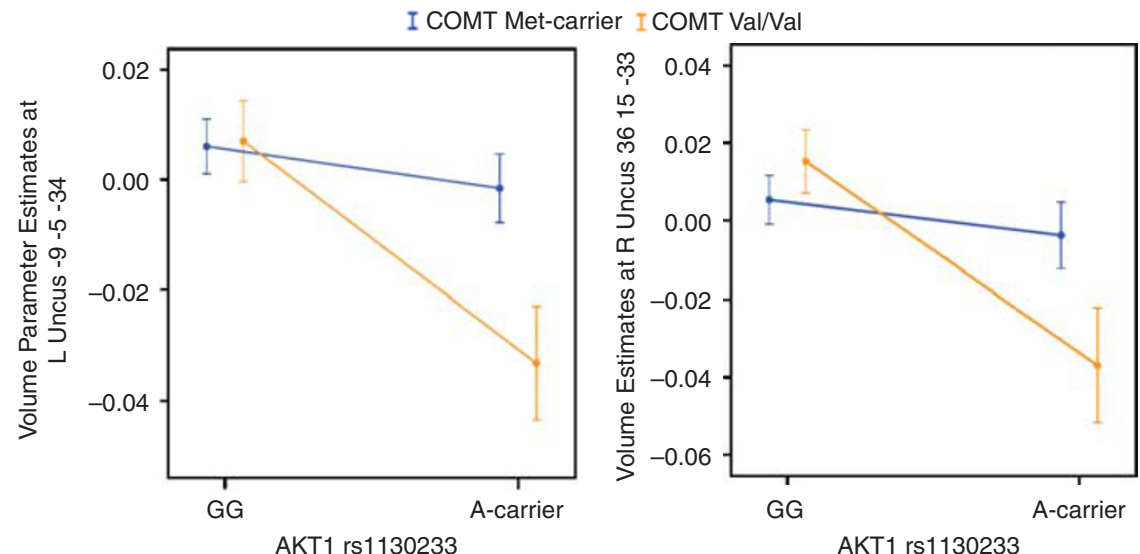

Figure 2 AKT1 and medial temporal lobe (MTL) gray-matter volume. (a) Main effects of AKT1 rs1130233 A-allele on reduced gray-matter volumes in left and right MTL regions $(n=171$; coordinates $-9-5-34, t=3.14$; coordinates $3615-33$, $t=3.10$, displayed at $P<0.001$ uncorrected). (b) Extracted parameter estimates from the left and right MTL peaks showing the main AKT1 effects also evidenced $A K T 1$-by-COMT interactions $(\mathrm{F}(1,162)=4.66, P=0.032$ and $\mathrm{F}(1,162)=4.69, P=0.032$ for the left and right loci, respectively). Individuals with combined $A K T 1$ A carriers and COMT-Val homozygotes ( $n=17)$ had disproportionately reduced gray-matter volumes at these loci. This set of results were permutation corrected $P<0.001$ within bilateral MTL regions-of-interest. Error bars are \pm 1 s.e.

empirical rate of falsely obtaining a similar set of main and 2-way interaction effects at these thresholds within the bilateral MTL regions-of-interest. The conservative rate at which this set of findings occurred was $P<0.0001$.

AKT1 epistatic interactions and risk for schizophrenia Genetic association with schizophrenia has been reported in multiple studies with each of these genes, but negative reports are virtually as frequent. As it is possible that the biologic epistasis between these genes that impacts on clinically relevant brain functions may also represent an epistatic mechanism of risk for clinical illness, we examined if the AKT1, $\mathrm{BDNF}$ and COMT genetic variations interacted to contribute to risk for schizophrenia. We first examined the case-control sample $(n=282$ patients, 329 controls) in which we previously reported marginal association with this AKT1 variant. ${ }^{11}$ While there were no marginal single gene associations for COMT or BDNF, the AKT1, COMT and BDNF variants showed a 3-way interaction $(P<0.041)$ in a full logistic regression model controlled for gender. The 3-way interaction was driven by a divergence of risk for schizophrenia in the context of the AKT1-A minor allele. Individuals at the highest risk were those who were also COMT-Val homozygotes and BDNF-Met carriers (Supplementary Results; Supplementary Figure S1).
While these directionally and biologically predictable interactions are unlikely to be by chance alone, the levels of statistical significance are small and would not survive genome-wide correction for multiple testing. Thus, we examined these relationships in an independent case-control sample (936 patients and 1190 controls) from the GAIN cohort (public release http://dbgap.ncbi.nlm.nih.gov/) for evidence of replication. There was the same 3-way AKT1, BDNF and COMT interaction on risk for schizophrenia (one-tailed $P=0.036$ ) and no significant individual gene main effects in a logistic regression model controlled for gender (Supplementary Results; Supplementary Figure S2). Permutation analysis revealed that the empirical likelihood that these same three variants would show the identical allelic direction of interactions in these two independent data sets is $P<7 \times 10^{-6}$.

Pharmacogenetic effects of AKT1 on cognition and brain structure in schizophrenia

Next, we explored AKT1 function in terms of clinically relevant pharmacology. As AKT1 impacted executive fronto-striatal cognition, and prefrontal structure and function, ${ }^{11,12}$ and additionally impacted key MTL processes as advanced here, we might expect that pharmacological modulation of AKT1 should affect a broad range of cognitive functions subserved by these regional effects and that these 
effects may show pharmacogenetic association if they relate to brain mechanisms in schizophrenia. We, therefore, examined interactions of clinically relevant pharmacological activators of AKT1-that is, lithium and sodium valproate ${ }^{32,33}$-and AKT1 genetic variation. We examined patients diagnosed with schizophrenia $(n=186)$ for whom we had complete treatment and cognitive data in terms of illness-associated reduction in IQ scores relative to premorbid IQ. This is a well-documented characteristic of patients with schizophrenia, ${ }^{36}$ is a reasonable proxy of general cognitive concomitants of illness and predicts treatment outcome and disability. ${ }^{37}$

Relative to schizophrenia patients on antipsychotics $(n=138)$, patients on antipsychotics and mood stabilizers (lithium and/or sodium Valproate, $n=48$ ) had higher chlorpromazine-equivalent antipsychotic doses (740(sd462) $\mathrm{mg}$ vs 528(sd421) mg, $t=2.95$, $P=0.003$ ) and more psychotic symptoms (PANSS positive: $\quad 15.7(\mathrm{sd} 7.0)$ vs $11.9(\mathrm{sd} 4.8), \quad t=3.72$, $P<0.001$; PANSS general: 29.1(sd10.0) vs 23.6(sd7.1), $t=3.72, P<0.001$; PANSS negative, n.s.). Patients on mood stabilizers also had marginally larger illness-associated cognitive change compared with those not receiving mood stabilizers (12.1(sd11.7) vs 8.4(sd11.2), $t=1.92, P=0.056)$. However, in patients treated with mood stabilizers, this cognitive change was not related to chlorpromazineequivalent antipsychotic dose, duration of illness, PANSS positive or negative symptoms, or gender (all $P>0.5)$. Instead, in a pharmacogenetic interaction, patients who carried the AKT1 rs1130233 A-allele associated with reduced AKT1 expression ${ }^{11,16,17}$ had relatively smaller cognitive change $(P=0.003 ; P<0.05$ Bonferroni corrected for the number of exploratory tests; Figure 3a) compared with non-risk allele carrier patients in the context of mood stabilizer treatment known to activate AKT1. ${ }^{32,33}$

Then, we examined a subset of these schizophrenia patients with available structural MRI data $(n=138)$ because previous studies have suggested that these mood stabilizing drugs affect gray-matter volume measures in patients treated with them. ${ }^{45}$ Using voxel-based morphometry, patients with the AKT1 A-allele who were also on mood stabilizers were found to have had regions in the MTL and prefrontal cortex that were disproportionately larger in graymatter volume compared with patients not receiving mood stabilizers in a pharmacogenetic interaction $(P<0.0001$ uncorrected; Figure 3b). Permutation tests showed that the combined rate of empirical falsepositive findings in terms of a similar set of pharmacogenetic interactions in cognition and interactions within regions-of-interest encompassing the bilateral MTL and prefrontal regions was $\mathrm{p}<0.0001$.

\section{Discussion}

We report human data that provide potential insights into the multiple levels through which AKT1 genetic variation may impact MTL brain processes relevant to human MTL development and cognitive function and to schizophrenia. Using functional and structural MRI phenotypes, we found that AKT1 genetic variation, through interactions with dopaminergic and neurotrophic processes referenced by COMT and BDNF, influenced brain correlates of neuroplasticity and development. The identical higher order allelic interactions also affected risk for schizophrenia in two independent clinical data sets. Further, AKT1 effects appear to have the potential for pharmacologic modification at least in schizophrenia patients.

The finding that the AKT1 rs1130233 variant influenced hippocampal memory function is consistent with AKT1 influence on short-term neuroplasticity associated with active memory encoding and implicit learning. ${ }^{22}$ This is further supported by AKT1 epistatic interactions with BDNF Val66Met and COMT Val158Met in the same hippocampal loci. In hippocampal neurons, there is critical molecular cross-talk between AKT and BDNF in enabling PSD95 dendritic trafficking, and consequent changes in N-methyl-D-aspartate (NMDA) and $\alpha$-amino-3hydroxy-5-methyl-4-isoxazolepropionic acid (AMPA) dynamics-these processes characterize long-term potentiation and may be key in active learning and memory processes. ${ }^{21,22}$ If so, the effects of AKT1 variation during active hippocampal memory processing should also be dependent on the efficacy of BDNF trafficking to precise dendritic sites of action, a process influenced by the BDNF Val66Met polymorphism. ${ }^{27,28,46}$ Our findings on human brain function are consistent with these possibilities. In addition, DA has an important modulatory role in memory function and hippocampal synaptic plasticity, ${ }^{25,26,31,40-44}$ as well as in BDNF dendritic processing in hippocampal neurons. ${ }^{39,47}$ This would be consistent with our 3-way interaction findings of accentuated AKT1 and BDNF coupled hippocampal memory dysfunction in the context of relatively compromised DA bioavailability indexed by the COMT-Val variant. Together, these data suggest that functional variants in these genes interact to influence hippocampal memory activity during short-term neuroplasticity. They also provide evidence linking the basic neuroscience of activity-dependent synaptic plasticity, ${ }^{21,22,25,39}$ and specific intermediate phenotypes implicated in the genetics of memory dysfunction in schizophrenia. ${ }^{14,48}$

For processes implicated in MTL gray-matter volume, our data suggest involvement of a partially overlapping set of genetic mechanisms. The AKT1related MTL structural changes observed appear to be less strongly linked to activity-dependent BDNF dendritic trafficking indexed by the Val66Met variation, and this AKT1-BDNF process is potentially more specific to active memory tasks rather than to MTL structure, at least as measured with MRI. The findings of AKT1 genetic effects on MTL structure are nevertheless consistent with the literature on the broad effects of AKT1 biology on brain structure, including putative effects on neuronal development, 


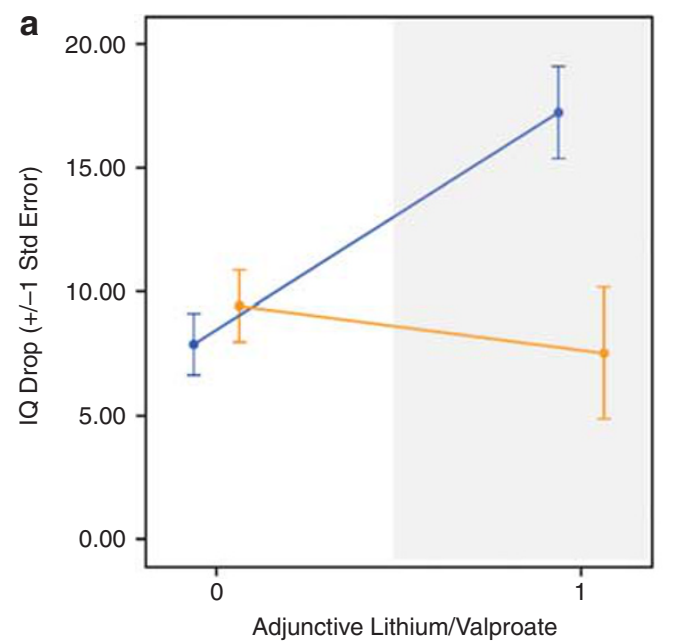

AKT1 Genotype

I $G G$

A-carrier

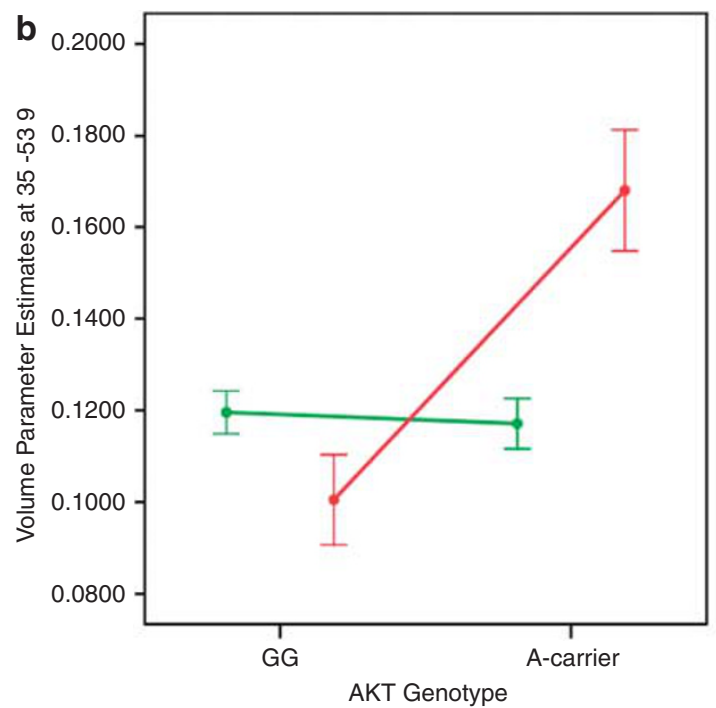

Lithium/valprote

I 0

I 1

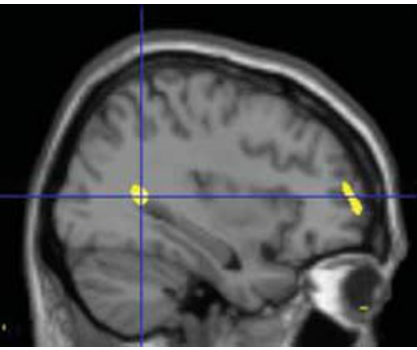

Figure 3 AKT1 pharmacogenetic interaction. (a) In the context of mood stabilizer treatment associated with AKT1 activation, schizophrenia patients who carried the AKT1 rs1130233 A-allele $(n=21)$ had relatively smaller cognitive change (total $n=186 ; \mathrm{F}(1,184)=9.06, P=0.003, P<0.05$ Bonferroni corrected for the number of exploratory tests). (b) Patients who were also on mood stabilizers were found to have had regions in the MTL and prefrontal cortex that were disproportionately larger in volume in relation to the AKT1 A-allele effect $(n=20)$, compared with patients not receiving mood stabilizers (total $n=138$; coordinates $35-539, \mathrm{~F}(1,130)=14.22, P<0.0001$ uncorrected; and coordinates $37592, \mathrm{~F}(1,130)=13.55, P<0.0001$ uncorrected, respectively). The set of pharmacogenetic results on cognition and brain were permutation corrected $P<0.0001$. Error bars were \pm 1 s.e.

myelination, actin polymerization and dendritic structure, $^{3}$ as well as AKT1 effects on neuroprotection $^{20}$ and its interactions with related drugs such as lithium and sodium valproate. ${ }^{32,33}$ Analogously, the interaction with DA is consistent with evidence that dendritic atrophy occurred in DA-depleted animal models. ${ }^{49}$ Changes in DA signaling through chronic opiate exposure reduced AKT signaling and decreased neuronal cell size. ${ }^{4}$ These observations are consistent with our findings that AKT1 genetic variation interacted with brain DA bioavailability indexed by the COMT variant to impact MTL gray-matter volume. Analogous structural brain findings, presumably through similar AKT1-dopaminergic effects on neurodevelopment, have also been demonstrated in human prefrontal cortex. ${ }^{11}$ Thus, the genetic control of AKT1-dopaminergic neurodevelopmental processes appeared to impact the prefrontal-MTL axis, and may coincide with known vulnerabilities of these brain structures in schizophrenia. $^{13}$

If combinations of genetic variants are ultimately implicated in disease mechanisms, we might expect some signature of these effects at the level of overall risk for the diagnosis of schizophrenia, even if these effects are small, given the complexities of the underlying processes they control. Moreover, epistatic models are increasingly recognized as critical for even simple behaviors in model systems and animals. ${ }^{50,51}$ Consistent with these possibilities, 
we found, in two independent case-control data sets, a 3-way interaction involving the functional AKT1, BDNF and COMT variants that affected risk for schizophrenia. Moreover, the directionalities of combinations of risk-associated alleles were consistent across data sets, where the combination of all three putatively disadvantageous alleles was associated with the highest schizophrenia risk. With the exception of a weak prior association with AKT1 A-alleles and schizophrenia in one of these data sets, ${ }^{11}$ main effects were not found for individual genes, consistent with other models for higher order epistasis in biological systems. ${ }^{50,51}$ The clinical associations add to the possibility that these genetic variants control relevant aspects of brain function associated with schizophrenia and that they do so as part of a diseaserelated biologic network. It has been proposed that multiple functional genetic variants layered across integrated biologic networks evolved toward stability of these pathways. ${ }^{52}$ For certain illnesses, genetic risk involving such pathways may require specific combinations of multiple variations which overwhelm compensatory processes and move the robust system away from its evolved optimum range. ${ }^{53}$

In further studying AKT1 variation and brain processes potentially related to schizophrenia, we examined in a clinical sample an hypothesis driven set of pharmacogenetic interactions with activators of AKT1 - that is, lithium or valproate ${ }^{32,33}$ - that are in common off-label use in patients with schizophrenia. It had previously been shown that AKT1 impacted executive fronto-striatal cognition, and prefrontal structure and function. ${ }^{11}$ If indeed AKT1 variation additionally impacted key MTL neurodevelopmental and plasticity processes as advanced here, we might then expect that pharmacological modulation of AKT1 should affect a range of cognitive functions in schizophrenia and that these effects would show genetic association. This was, in fact, what we found, using a well-validated measure of illness-related cognitive change. ${ }^{36}$ Individuals who had the AKT1 risk variant associated with relatively reduced AKT1 expression and deleterious prefrontal-MTL brain effects ${ }^{11,16,17}$ appeared to have relatively less cognitive deterioration if they were on activators of AKT1, lithium or sodium valproate, compared with non-risk allele carriers. Correspondingly, these individuals had regions in the prefrontal cortex and MTL with relatively increased gray-matter volumes. Our results are consistent with previous studies that have suggested that these drugs lead to gray-matter volume increases in patients receiving them. ${ }^{45}$ Our pharmacogenetic results offer the suggestion that some AKT1 brain effects could potentially be pharmacologically modifiable and genotypically variable.

Nevertheless, the interpretation of our pharmacogenetic data is problematic. While clinical experience suggests that schizophrenia patients who are more ill tend to be treated with adjunctive lithium or valproate ${ }^{54}$ future prospective studies will be needed to disambiguate the alternative possibilities that these treatments could have contributed to the worse symptomatic and cognitive outcome in the AKT1 G homozygotes, or that there was improvement (or less deterioration) of cognitive and brain effects in the AKT1 A-carrier patients potentially from resilience to drug-related side effects in these individuals. We also did not study blood levels of these drugs, which should be noted in future work. However, the pharmacogenetic finding remains that an over-representation of patients on additional AKT1 activating drugs, lithium or valproate, had differing cognitive and prefrontal-MTL brain outcomes determined by AKT1 genetic variation. This targeted pharmacogenetic finding extends key predictions from the imaging effects of AKT1 variation in healthy individuals reported here and previously. ${ }^{11,12,18}$ The pharmacogenetic effect on cognition was independent of confounds from symptoms and antipsychotic drugs. However, our study was not powered to explore associations between AKT1 and other clinical or symptom variables for which we had no prior hypothesis or imaging data. Indeed, it bears noting that it was in the patients receiving mood stabilizers that the AKT1 effects on cognition and brain were focused. In the entire sample, there was no overall effect of AKT1 on disease-related cognitive decline. These findings are consistent with a modulatory cognitive-brain effect of AKT1 activation by mood stabilizers in some patients with the deleterious AKT1 A-allele and receiving these drugs.

In conclusion, we have reported a convergent series of human studies identifying effects of AKT1 variation in memory-dependent neuroplasticity and structural brain processes relevant to normal brain development and to schizophrenia, and epistatic interactions with neurotrophic and dopaminergic processes that modulate these AKT1 effects. Pharmacologic modulation of AKT1 was further associated with effects on disease-related cognition and corresponding prefrontal-MTL brain structure in schizophrenia patients. AKT1 and related neuroplasticity and developmental pathways may, therefore, genetically influence cognition and risk for schizophrenia through effects that may be pharmacologically modifiable.

\section{Conflict of interest}

The authors declare no conflict of interest.

\section{Acknowledgments}

This work was funded by the National Institute of Mental Health Intramural Research Program.

\section{References}

1 Lai WS, Xu B, Westphal KGC, Paterlini M, Olivier B, Pavlidis P et al. Akt1 deficiency affects neuronal morphology and predisposes to abnormalities in prefrontal cortex functioning. Proc Natl Acad Sci USA 2006; 103: 16906-16911. 
2 Beaulieu J-M, Gainetdinov RR, Caron MG. The Akt-GSK-3 signaling cascade in the actions of dopamine. Trends Pharmacol Sci 2007; 28: 166.

3 Lai WS, Xu B, Westphal KGC, Paterlini M, Olivier B, Pavlidis P et al. Akt1 deficiency affects neuronal morphology and predisposes to abnormalities in prefrontal cortex functioning. Proc Natl Acad Sci USA 2006; 103: 16906-16911.

4 Russo SJ, Bolanos CA, Theobald DE, DeCarolis NA, Renthal W, Kumar A et al. IRS2-Akt pathway in midbrain dopamine neurons regulates behavioral and cellular responses to opiates. Nat Neurosci 2007; 10: 93-99.

5 Levitt P, Harvey JA, Friedman E, Simansky K, Murphy EH. New evidence for neurotransmitter influences on brain development. Trends Neurosci 1997; 20: 269.

6 Li Z, Jaboin J, Dennis PA, Thiele CJ. Genetic and pharmacologic identification of Akt as a mediator of brain-derived neurotrophic factor/TrkB rescue of neuroblastoma cells from chemotherapyinduced cell death. Cancer Res 2005; 65: 2070-2075.

7 Schwab SG, Hoefgen B, Hanses C, Hassenbach MB, Albus M, Lerer $\mathrm{B}$ et al. Further evidence for association of variants in the AKT1 gene with schizophrenia in a sample of European sib-pair families. Biol Psychiatry 2005; 58: 446.

8 Thiselton DL, Vladimirov VI, Kuo P-H, McClay J, Wormley B, Fanous A et al. AKT1 is associated with schizophrenia across multiple symptom dimensions in the Irish study of high density schizophrenia families. Biol Psychiatry 2007; 63: 449-457.

9 Emamian ES, Hall D, Birnbarum MJ, Karayiogou M, Gogos JA. Convergent evidence for impaired AKT1-GSK3b signaling in schizophrenia. Nat Genet 2004; 36: 131-137.

10 Norton N, Williams HJ, Dwyer S, Carroll L, Peirce T, Moskvina V et al. Association analysis of AKT1 and schizophrenia in a UK case control sample. Schizophr Res 2007; 93: 58-65.

11 Tan HY, Nicodemus KK, Chen Q, Li Z, Honea R, Brooke J et al. Genetic variation in AKT1 is linked to dopamine-associated prefrontal cortical structure and function in humans. J Clin Invest 2008; 118: 2200-2208.

12 Blasi G, Napolitano F, Ursini G, Taurisano P, Romano R, Caforio G et al. DRD2/AKT1 interaction on D2 c-AMP independent signaling, attentional processing, and response to olanzapine treatment in schizophrenia. Proc Natl Acad Sci USA 2011; 108: 1158-1163.

13 Lipska BK, Jaskiw GE, Weinberger DR. Postpubertal emergence of hyperresponsiveness to stress and to amphetamine after neonatal excitotoxic hippocampal damage: A potential animal model of schizophrenia. Neuropsychopharmacology 1993; 9: 67-75.

14 Harrison PJ, Weinberger DR. Schizophrenia genes, gene expression, and neuropathology: on the matter of their convergence. Mol Psychiatry 2005; 10: 40-68.

15 Weinberger DR, Egan MF, Bertolino A, Callicott JH, Mattay VS, Lipska BK et al. Prefrontal neurons and the genetics of schizophrenia. Biol Psychiatry 2001; 50: 825-844.

16 Harris SL, Gil G, Robins H, Hu W, Hirshfield K, Bond E et al. Detection of functional single-nucleotide polymorphisms that affect apoptosis. Proc Natl Acad Sci USA 2005; 102: 16297-16302.

17 Giovannetti E, Zucali PA, Peters GJ, Cortesi F, D'Incecco A, Smit $\mathrm{EF}$ et al. Association of polymorphisms in AKT1 and EGFR with clinical outcome and toxicity in non-small cell lung cancer patients treated with gefitinib. Mol Cancer Ther 2010; 9: 581-593.

18 Olli PHP, Tiina P, Anu L, Annamari T-H, Tuula K, Paul T et al. Association of AKT1 with verbal learning, verbal memory, and regional cortical gray matter density in twins. Am J Med Genet B Neuropsychiatr Genet 2009; 150B: 683-692.

19 Beaulieu JM, Sotnikova TD, Marion S, Lefkowitz RJ, Gainetdinov RR, Caron MG. An Akt/ $\beta$-Arrestin 2/PP2A signaling complex mediates dopaminergic neurotransmission and behavior. Cell 2005; 122: 261-273.

20 Johnson-Farley NN, Travkina T, Cowen DS. Cumulative activation of Akt and consequent inhibition of glycogen synthase kinase-3 by brain-derived neurotrophic factor and insulin-like growth factor-1 in cultured hippocampal neurons. J Pharmacol Exp Ther 2006; 316: 1062-1069.

21 Spencer JL, Waters EM, Milner TA, Lee FS, McEwen BS. BDNF variant Val66Met interacts with estrous cycle in the control of hippocampal function. Proc Natl Acad Sci USA 2010; 107: $4395-4400$.
22 Yoshii A, Constantine-Paton M. BDNF induces transport of PSD95 to dendrites through PI3K-AKT signaling after NMDA receptor activation. Nat Neurosci 2007; 10: 702.

23 Weickert CS, Hyde TM, Lipska BK, Herman MM, Weinberger DR, Kleinman JE. Reduced brain-derived neurotrophic factor in prefrontal cortex of patients with schizophrenia. Mol Psychiatry 2003; 8: 592.

24 Glorioso C, Sabatini M, Unger T, Hashimoto T, Monteggia LM, Lewis DA et al. Specificity and timing of neocortical transcriptome changes in response to BDNF gene ablation during embryogenesis or adulthood. Mol Psychiatry 2006; 11: 633-648.

25 Huang YY, Kandel ER. D1/D5 receptor agonists induce a protein synthesis-dependent late potentiation in the CA1 region of the hippocampus. Proc Natl Acad Sci USA 1995; 92: 2446-2450.

26 Lisman JE, Grace AA. The Hippocampal-VTA Loop: controlling the entry of information into long-term memory. Neuron 2005; 46 703.

27 Egan MF, Kojima M, Callicott JH, Goldberg TE, Kolachana BS, Bertolino A et al. The BDNF val66met polymorphism affects activity-dependent secretion of BDNF and human memory and hippocampal function. Cell 2003; 112: 257.

28 Chen Z-Y, Jing D, Bath KG, Ieraci A, Khan T, Siao C-J et al. Genetic variant BDNF (Val66Met) polymorphism alters anxiety-related behavior. Science 2006; 314: 140-143.

29 Chen J, Lipska BK, Halim N, Ma QD, Matsumoto M, Melhem S et al. Functional analysis of genetic variation in catechol-Omethyltransferase (COMT): effects on mRNA, protein, and enzyme activity in postmortem human brain. Am I Hum Genet 2004; 75 807-821.

30 Egan MF, Goldberg TE, Kolachana BS, Callicott JH, Mazzanti CM, Straub RE et al. Effect of COMT Val108/158 Met genotype on frontal lobe function and risk for schizophrenia. Proc Natl Acad Sci USA 2001; 98: 6917-6922.

31 Slifstein M, Kolachana B, Simpson EH, Tabares P, Cheng B, Duvall $\mathrm{M}$ et al. COMT genotype predicts cortical-limbic D1 receptor availability measured with 11C NNC112 and PET. Mol Psychiatry 2008; 13: 821-827.

32 Chalecka-Franaszek E, Chuang DM. Lithium activates the serine/ threonine kinase Akt-1 and suppresses glutamate-induced inhibition of Akt-1 activity in neurons. Proc Natl Acad Sci USA 1999; 96: 8745 .

33 De Sarno P, Li X, Jope RS. Regulation of Akt and glycogen synthase kinase-3 beta phosphorylation by sodium valproate and lithium. Neuropharmacology 2002; 43: 1158.

34 Hariri AR, Goldberg TE, Mattay VS, Kolachana BS, Callicott JH, Egan MF et al. Brain-derived neurotrophic factor val66met polymorphism affects human memory-related hippocampal activity and predicts memory performance. I Neurosci 2003; 23: 6690-6694.

35 Pezawas L, Verchinski BA, Mattay VS, Callicott JH, Kolachana BS, Straub RE et al. The brain-derived neurotrophic factor val66me polymorphism and variation in human cortical morphology. J Neurosci 2004; 24: 10099-10102.

36 Weickert TW, Goldberg TE, Gold JM, Bigelow LB, Egan MF Weinberger DR. Cognitive impairments in patients with schizophrenia displaying preserved and compromised intellect. Arch Gen Psychiatry 2000; 57: 907.

37 Green MF. What are the functional consequences of neurocognitive deficits in schizophrenia? Am J Psychiatry 1996; 153. 321-330.

38 Genovese CR, Lazar NA, Nichols T. Thresholding of statistical maps in functional imaging using the false discovery rate. Neuroimage 2002; 15: 870-878.

39 Ji Y, Pang PT, Feng L, Lu B. Cyclic AMP controls BDNF-induced TrkB phosphorylation and dendritic spine formation in mature hippocampal neurons. Nat Neurosci 2005; 8: 164

40 Jay TM. Dopamine: a potential substrate for synaptic plasticity and memory mechanisms. Prog Neurobiol 2003; 69: 375-390.

41 Schott BH, Seidenbecher CI, Fenker DB, Lauer CJ, Bunzeck N, Bernstein $\mathrm{H}-\mathrm{G}$ et al. The dopaminergic midbrain participates in human episodic memory formation: evidence from genetic imaging. I Neurosci 2006; 26: 1407-1417.

42 Bertolino A, Di Giorgio A, Blasi G, Sambataro F, Caforio G, Sinibaldi L et al. Epistasis between dopamine regulating genes 
identifies a nonlinear response of the human hippocampus during memory tasks. Biol Psychiatry 2008; 64: 226-234.

43 Bertolino A, Rubino V, Sambataro F, Blasi G, Latorre V, Fazio L et al. Prefrontal-hippocampal coupling during memory processing is modulated by COMT Val158Met genotype. Biol Psychiatry 2006; 60: $1250-1258$.

44 Krach S, Jansen A, Krug A, Markov V, Thimm M, Sheldrick AJ et al. COMT genotype and its role on hippocampal-prefrontal regions in declarative memory. Neuroimage 2010; 53: 978-984.

45 Bearden CE, Thompson PM, Dutton RA, Frey BN, Peluso MAM, Nicoletti $\mathrm{M}$ et al. Three-dimensional mapping of hippocampal anatomy in unmedicated and lithium-treated patients with bipolar disorder. Neuropsychopharmacology 2007; 33: 1229.

46 Soliman F, Glatt CE, Bath KG, Levita L, Jones RM, Pattwell SS et al. A genetic variant BDNF polymorphism alters extinction learning in both mouse and human. Science 2010; 327: 863-866.

47 Rossato JI, Bevilaqua LRM, Izquierdo I, Medina JH, Cammarota M. Dopamine controls persistence of long-term memory storage. Science 2009; 325: 1017-1020.

48 Skelley SL, Goldberg TE, Egan MF, Weinberger DR, Gold JM. Verbal and visual memory: characterizing the clinical and intermediate phenotype in schizophrenia. Schizophr Res 2008; 105: 78.

49 Sherren N, Pappas BA. Selective acetylcholine and dopamine lesions in neonatal rats produce distinct patterns of cortical dendritic atrophy in adulthood. Neuroscience 2005; 136: $445-456$.

50 Shao H, Burrage LC, Sinasac DS, Hill AE, Ernest SR, O'Brien W et al. Genetic architecture of complex traits: large phenotypic effects and pervasive epistasis. Proc Natl Acad Sci USA 2008; 105: 19910-19914.

51 Gerke J, Lorenz K, Cohen B. Genetic interactions between transcription factors cause natural variation in yeast. Science 2009; 323: 498-501.

52 Waddington $\mathrm{CH}$. Canalization of development and the inheritance of acquired characters. Nature 1942; 150: 563.

53 Moore JH, Williams SM. Epistasis and its implications for personal genetics. Am J Human Genet 2009; 85: 309.

54 Fountoulakis KN, Vieta E, Bouras C, Notaridis G, Giannakopoulos $\mathrm{P}$, Kaprinis $\mathrm{G}$ et al. A systematic review of existing data on longterm lithium therapy: neuroprotective or neurotoxic? Int $J$ Neuropsychopharmacol 2008; 11: 269.

(c) This work is licensed under the Creative Commons Attribution-NonCommercialNo Derivative Works 3.0 Unported License. To view a copy of this license, visit http://creativecommons. org/licenses/by-nc-nd/3.0/

Supplementary Information accompanies the paper on the Molecular Psychiatry website (http://www.nature.com/mp) 